\title{
BMJ Open Key elements of effective postgraduate GP educational environments: a mixed methods study
}

\author{
Bunmi S. Malau-Aduli (D), Faith O. Alele (1) , Paula Heggarty, Carole Reeve, \\ Peta-Ann Teague
}

To cite: Malau-Aduli BS, Alele F0, Heggarty P, et al. Key elements of effective postgraduate GP educational environments: a mixed methods study. BMJ Open 2021;11:e041110. doi:10.1136/ bmjopen-2020-041110

- Prepublication history and additional material for this paper is available online. To view these files, please visit the journal online (http://dx.doi.org/10. 1136/bmjopen-2020-041110)

Received 30 May 2020 Revised 14 November 2020 Accepted 22 December 2020

Check for updates

(c) Author(s) (or their employer(s)) 2021. Re-use permitted under CC BY-NC. No commercial re-use. See rights and permissions. Published by BMJ.

College of Medicine and Dentistry, James Cook University, Townsville, Queensland, Australia

Correspondence to A/Prof Bunmi S. Malau-Aduli; bunmi.malauaduli@jcu.edu.au

\section{ABSTRACT}

Objectives Evidence in the literature suggests that satisfaction with postgraduate general practice (GP) training is associated with the quality of the educational environment. This study aimed to examine GP registrars' level of satisfaction with a distributed model of training in a regional educational environment and investigate the relationship between satisfaction and academic performance.

Study design A longitudinal 3-year study was conducted among GP registrars at James Cook University using a sequential explanatory mixed methods research design. GP registrars' satisfaction was obtained using the scan of postgraduate educational environment domains tool. A focus group discussion was conducted to explore GP registrars' perceptions of satisfaction with the educational environment.

Setting James Cook University General Practice Training (JCU GPT) programme.

Participants Six hundred and fifty one (651) GP registrars enrolled between 2016 and 2018 at JCU GPT programme. Results 651 registrars completed the satisfaction survey between 2016 and 2018 . Overall, $92 \%$ of the registrars were satisfied with the educational training environment. Registrars who had become fellows reported higher satisfaction levels compared with those who were still in training (mean $=4.39$ vs $4.20, p=0.001$ ). However, academic performance had no impact on level of satisfaction with the educational environment. Similarly, practice location did not influence registrars' satisfaction rates. Four themes (rich rural/remote educational environment, supportive learning environment, readiness to continue with rural practice and practice culture) emerged from the thematic data analysis.

Conclusion A clinical learning environment that focuses on and supports individual learning needs is vital for effective postgraduate medical training. This study suggests that JCU GPT programme's distributed model fostered a satisfying and supportive training environment with rich educational experiences that enhance retention of GP registrars in rural/remote North Queensland, Australia. The findings of this study may be applicable to other settings with similar training models.

\section{BACKGROUND}

International evidence reveals that primary healthcare is an integral part of the health

\section{Strengths and limitations of this study}

This study is the first to investigate GP registrars satisfaction with the clinical educational environment that uses a distributed training model.

- This study employed a mixed methods research design that gives credence to the study findings because they are grounded in participants' experiences.

- This study was conducted among GP registrars in one training organisation in rural/remote region in Australia.

- The findings of this study need to be interpreted with caution when applied to other postgraduate training models.

system and is key to improving health outcomes. ${ }^{1}$ However, the provision of adequate and accessible primary healthcare services for populations in rural and remote locations has been a challenge for governments across the globe. ${ }^{2}$ The impact is quite significant for populations residing in rural and remote Australia who experience poorer health including a life expectancy at birth that is 10 years less than the general population in metropolitan locations. ${ }^{3}$ These poorer health outcomes are associated with geographical remoteness and maldistribution of primary healthcare providers. ${ }^{4}$ For primary care services to be more effective in delivering quality healthcare, improving health outcomes and reducing disparities, it is important that care providers are evenly distributed across diverse population groups. ${ }^{5}$

For decades, rural and remote regions in Australia have encountered varied challenges with respect to medical workforce recruitment and retention. ${ }^{5}$ Strategies used to address these challenges include providing incentives to take up practice in rural and remote regions, providing increased access to allied health services and increasing the number of training places for doctors 
in general practice (GP). ${ }^{5}$ In Australia, GP training is organised by the Australian General Practice Training programme (AGPT). The training programme involves a minimum of 3 years' commitment, and the general practitioners (GPs) in training work under supervision in accredited training practices. ${ }^{67}$ More recently, the James Cook University (JCU) General Practice Training (GPT) programme was established in 2016 to increase the workforce in rural and remote Northern Queensland. ${ }^{8}$ The JCU GPT programme is unique in that it adopts a distributed model in the delivery of its educational training programme. Given that the JCU GPT programme is relatively new (distributed model) and solely targeted at training in rural and remote regions, it is important to assess its impact on GP registrars' satisfaction with their training.

Satisfaction in postgraduate GP training is associated with the quality of the educational environment. ${ }^{9}$ In addition, person-environment fit has been shown to be a predictor of satisfaction and academic performance. ${ }^{10}$ Evidence suggests that doctors' perceptions of satisfaction with the work environment contribute to retention, quality of care and patient satisfaction. ${ }^{11-17}$ A number of factors that have been shown to increase job satisfaction include professional autonomy, academic involvement, role modelling, freedom in work management and organisation of working environment, intellectual stimulation and relationship with colleagues. ${ }^{11-17}$ Determinants of poor job satisfaction include heavy workload, time constraints, dissatisfaction with training, administrative burdens and low income. ${ }^{11} 121415$ Nonetheless, studies that have investigated the impact of practice location on job satisfaction among GPs are inconclusive. While some studies suggest that GPs working in rural areas have higher levels of satisfaction, ${ }^{12} 18$ other studies have reported that GPs whose practices are located in urban cities are more likely to be satisfied with their professional lives than their counterparts who work in rural locations. ${ }^{11}$ Furthermore, other evidence suggest that there is no association between job satisfaction levels and remoteness. ${ }^{19-21}$

The JCU GPT programme provides training for registrars across different rural and remote communities and places emphasis on individualised support for the registrars,including access to a training supervisor per registrar, resources, community-based infrastructure and review of progress at regular intervals. ${ }^{22}{ }^{23}$ According to Peel $e t a l^{23}$, the JCU GPT localised training mitigated issues (such as social and professional isolation and accessing professional development) associated with rural and remote practice. It was reported that the localised training programme increased the attractors to rural and remote practice and fostered community engagement and professional network. ${ }^{23}$ It is believed that this localised training programme with its unique model of individualised support can promote and improve the recruitment and retention of GPs in underserved/ remote areas. ${ }^{23}$ Given that job satisfaction is a significant predictor of retention, ${ }^{14}$ it is important to investigate the level of satisfaction and perceptions of the registrars concerning the localised training model.

Therefore, this study examined JCU GP registrars' satisfaction with the educational environment and their perceptions of the work/educational environment. The study also examined the association between the registrars' level of satisfaction with the educational environment and their academic performance in the Royal Australian College of General Practice (RACGP) and Australian College of Rural and Remote Medicine (ACRRM) GP specialty training examinations.

\section{METHODS}

A sequential explanatory mixed methods design was employed using a pragmatic paradigm in this 3-year (2016, 2017 and 2018) study. ${ }^{24}$ A sequential explanatory approach consists of two phases in which quantitative data are first collected and analysed, followed by collection and analyses of qualitative data, and explanation of the quantitative data using the qualitative results. ${ }^{26} \mathrm{~A}$ pragmatic paradigm embraces both quantitative and qualitative approach and offers a flexible and more reflexive approach to investigate a phenomenon and address research questions. ${ }^{25}$ Given that pragmatism is associated with abductive reasoning that alternates between deduction and induction, we aimed to provide an insightful construct/reason to explain the relationship between satisfaction and perception of the educational environment. ${ }^{25}$ It is important to note that this study focused on the first two levels (reaction and learning) of Kirkpatrick's Training Evaluation model. ${ }^{27}$

\section{First phase: quantitative}

At the end of each academic year, all enrolled JCU GPT registrars were invited to express their level of satisfaction with the educational environment by completing a 15-item validated postgraduate educational environment survey tool: scan of postgraduate educational environment domains (SPEED) and an open-ended question about their perceptions of the educational/training environment. Details of the validity of the SPEED tool in assessing the quality of the educational environment in a rural GP training setting has been documented in a previously published article. ${ }^{28}$ This current study is a follow-up longitudinal study that focuses on the factors that influence the registrars' satisfaction with their clinical educational environment. To determine the association between satisfaction levels and academic performance, participants' results in the GP college examinations were extracted from the systems database and correlated with their perceived satisfaction rates.

\section{Second phase: qualitative}

Data for the qualitative phase of the study comprised the open-ended responses from the survey and focus group discussion (FGD). Semistructured open-ended questions were used in the FGD to further explore the 
perceptions of the registrars about the clinical learning environment. Using convenience sampling, participants were recruited into the FGD, participation was voluntary and no incentives were offered to the participants. The semistructured interview guide was developed by the research team and pilot-tested before use. The questions used in the FGD were designed to facilitate discussion and included the following: (1) how would you generally describe your GP training experience?; (2) what are the advantages of training in your current location?; (3) how do you feel or perceive your GP training might have been disadvantaged by training in your current location, as opposed to somewhere else?; and (4) what was the most valuable support or input provided to you in this practice location? Two experienced independent researchers who were not involved in the training/ supervision of the participants conducted the FGD. The research team discussed the interview questions and the broad aims of the study with the interviewers before the FGD was conducted. The FGD was audio-recorded and lasted for approximately $40 \mathrm{~min}$. Discussions within the FGD continued until data saturation was achieved; that is, the participants' responses were no longer revealing new information. ${ }^{29}$

\section{Study context}

The JCU GPT programme (formerly known as Generalist Medical Training) was established by JCU in 2016 as part of the GP training pipeline in response to the need for GP training in regional, rural and remote areas. ${ }^{8}$ The distributed model provides training for registrars in more than 350 training posts across smaller rural and remote communities in central, northern and western Queensland. Overall, the training model ensures that the registrars are supported in practice by clinical supervisors and regionally based administrative and medical educator staff in each of the training sites (figure 1). Each registrar is provided with individualised support that includes provision of a training supervisor who is designated with the responsibility of mapping their training pathway and reviewing progress and curriculum and assessment issues at regular intervals for each registrar. ${ }^{22}{ }^{23}$ In addition, a designated administration staff member at the regional training node provides administrative support to the

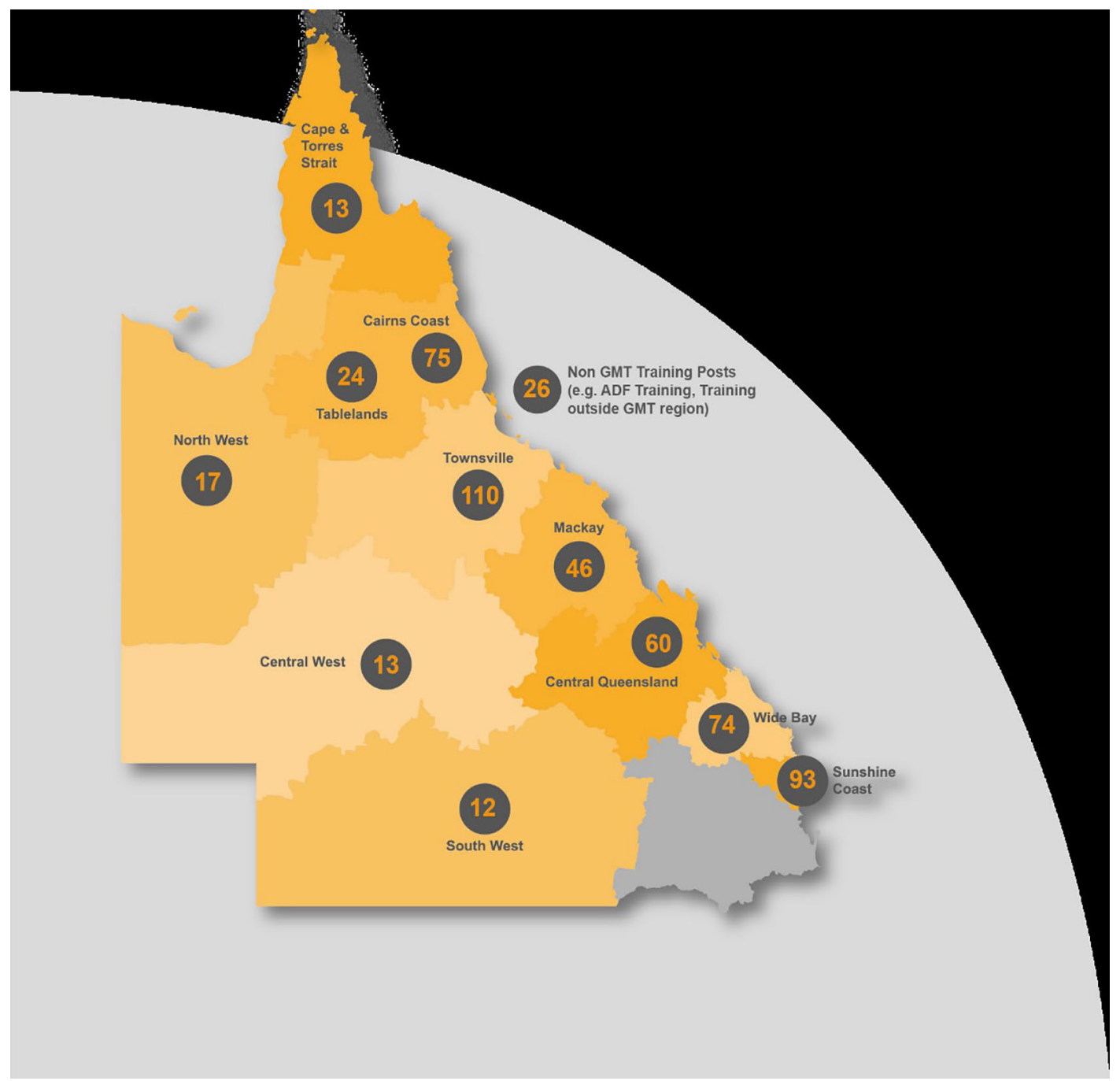

Figure 1 Geographical distribution of registrars in JCU GPT regions. 
registrars. ${ }^{9}{ }^{10}$ The JCU GPT is a 3-year to 4-year full-time training programme depending on the choice of fellowship: RACGP or ACRRM.

\section{Patient and public involvement}

Patients and the public were not involved in the planning or design of this study.

\section{Statistical analyses}

The quantitative data were analysed using SPSS V.25. The effects of type of fellowship, college and remoteness on satisfaction scores were determined using Mann-Whitney $\mathrm{U}$ test and Kruskal-Wallis $\mathrm{H}$ test, respectively. Further analysis was conducted to assess the relationship between satisfaction rates and pass/fail rates in the fellowship examinations using $\chi^{2}$ test for independence. To maximise available sample size for the analysis, missing data were deleted pairwise. The level of significance was set at $\mathrm{p}<0.05$.

The qualitative (FGD and the open-ended survey responses) data were fully transcribed by an independent professional transcriber. The transcribed and deidentified data were thematically analysed in NVivo V.12 analytical software. The steps described by Braun and Clarke were used for the thematic analysis ${ }^{30}:(1)$ data were read several times to ensure familiarisation and to get an overall impression of the content, (2) patterns were identified and organised into codes, (3) emerging themes were generated from the identified codes, (4) the initial themes were reviewed and grouped, (5) the identified themes were refined and (6) representative quotes were selected to support each theme. The first coding and generation of themes was conducted by FA. To ensure credibility and trustworthiness, the transcribed data, codes and themes were reviewed independently by BM-A. Data were discussed and reviewed in a consensus meeting by the authors, and there was approximately $95 \%$ degree of congruence between both authors' codes, themes and classifications. Discrepancies were discussed until mutual agreement was reached. The other authors cross-checked the quotes and themes to ensure consistency. The qualitative part of the study adhered to the standards for reporting qualitative research guidelines (online supplemental file 1). ${ }^{31}$ The findings of the qualitative data were used to explain the results of the quantitative part of the study (triangulation) to increase the rigour and validity of this research.

\section{RESULTS}

Six hundred and fifty-one (651) registrars completed the satisfaction survey between 2016 and 2018. The mean age of the respondents was $35.32 \pm 6.44$ years, and more than $60 \%$ of the registrars were female (table 1). Over $80 \%$ of the registrars were undertaking their training in regional areas (inner and outer regional). In terms of fellowship examination, 322 registrars had attempted the fellowship examination during the periods considered in this study.

\begin{tabular}{|c|c|c|}
\hline Characteristics & Frequency & Percentage \\
\hline \multicolumn{3}{|l|}{ Age (years) } \\
\hline 25-29 & 113 & 17.4 \\
\hline $30-34$ & 232 & 35.6 \\
\hline $35-39$ & 157 & 24.1 \\
\hline 40 and above & 149 & 22.9 \\
\hline \multicolumn{3}{|l|}{ Gender } \\
\hline Male & 239 & 36.7 \\
\hline Female & 412 & 63.3 \\
\hline \multicolumn{3}{|l|}{ Graduate status } \\
\hline $\begin{array}{l}\text { Australian medical } \\
\text { graduate }\end{array}$ & 439 & 67.4 \\
\hline $\begin{array}{l}\text { International medical } \\
\text { graduate }\end{array}$ & 212 & 32.6 \\
\hline \multicolumn{3}{|c|}{ Aboriginal and Torres Strait Islander status } \\
\hline Yes & 9 & 1.4 \\
\hline No & 642 & 98.6 \\
\hline \multicolumn{3}{|l|}{ College } \\
\hline RACGP & 485 & 74.5 \\
\hline ACRRM & 141 & 21.7 \\
\hline RACGP and ACRRM & 25 & 3.8 \\
\hline \multicolumn{3}{|l|}{ Fellowship status } \\
\hline Fellowed & 221 & 33.9 \\
\hline Not fellowed & 430 & 66.1 \\
\hline \multicolumn{3}{|c|}{ Remoteness area (RA) classification of training location } \\
\hline Inner regional ${ }^{*}$ & 312 & 48.1 \\
\hline Outer regional & 281 & 43.4 \\
\hline Remote Australia & 38 & 5.9 \\
\hline Very remote Australia & 17 & 2.6 \\
\hline \multicolumn{3}{|l|}{ Year of study } \\
\hline 2016 & 183 & 28.1 \\
\hline 2017 & 280 & 43.0 \\
\hline 2018 & 188 & 28.9 \\
\hline
\end{tabular}

${ }^{*} \mathrm{~A}$ small proportion of the registrars were located in regions coded as major cities. For the purpose of analysis and to ensure data reliability, data for registrars in major cities and those in inner regional areas were clustered.

ACRRM, Australian College of Rural and Remote Medicine; RACGP, Royal Australian College of General Practice.

Overall, $92 \%$ of registrars were satisfied with the educational training environment (figure 2). There was no significant difference in the level of satisfaction in relation to the study period with approximately $92 \%, 93 \%$ and $91 \%$, respectively, satisfied with the educational training environment over the 3-year period $\left(\chi^{2}=7.803, \mathrm{p}=0.277\right)$.

There was no significant difference in satisfaction level in relation to college affiliations. Registrars enrolled in the RACGP pathway had similar satisfaction rates to registrars enrolled in ACRRM as well as those who were enrolled 


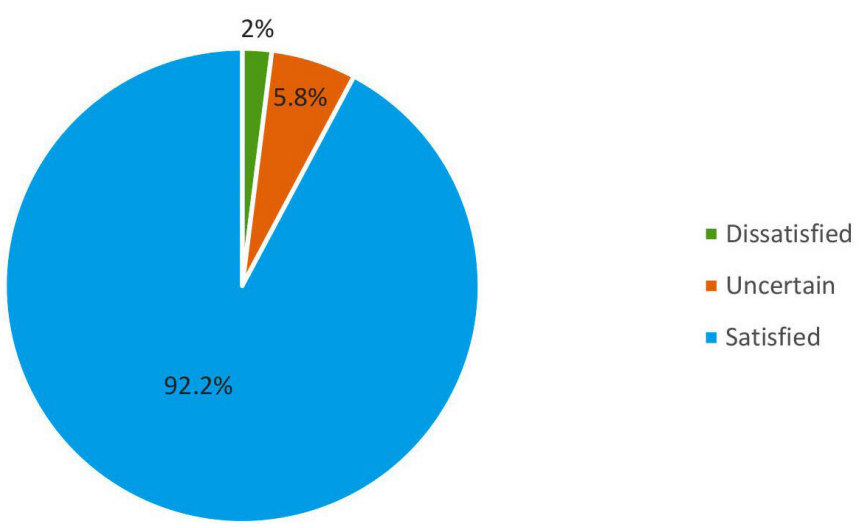

Figure 2 Registrars' overall satisfaction with the training environment.

in both ACRRM and RACGP (median=4, Kruskal-Wallis $\mathrm{H}=5.082, \mathrm{df}=2, \mathrm{p}=0.079$ ).

There was a significant difference in overall satisfaction between participants who had fellowed and those still in training. The fellows reported a higher satisfaction rate compared with the registrars still in training (median $=4$, Mann-Whitney $\mathrm{U}=40909.500$, $\mathrm{p}=0.001$ ).

There was no significant difference in overall registrars' satisfaction rates in relation to practice location (KruskalWallis $\mathrm{H}=1.765$, $\mathrm{df}=3, \mathrm{p}=0.623$ ). The mean overall satisfaction score for registrars located in inner regional areas and outer regional areas were $4.27(\mathrm{SD}=0.61$, median $=4, \mathrm{IQR}=1)$ and $4.28 \quad(\mathrm{SD}=0.7$, median $=4, \mathrm{IQR}=1)$, respectively. The mean overall satisfaction score for registrars located in remote and very remote Australia were $4.08(\mathrm{SD}=0.8$, median $=4, \mathrm{IQR}=1)$ and $4.29(\mathrm{SD}=0.47$, median $=4, \mathrm{IQR}=1)$.

\section{Association between satisfaction rate and performance on college exams}

Three hundred and twenty-two (322) registrars had written the fellowship examinations during this study, and 51.3\% of those who sat the exams had become fellows of the GP Colleges. Overall, $75.4 \%$ of the registrars who attempted the exam passed the RACGP examination, while $24.6 \%$ failed. However, passing the RACGP fellowship examination was independent of satisfaction with the training environment $\left(\chi^{2}=0.936, \mathrm{df}=2, \mathrm{p}=0.626\right)$.

Similarly, among the registrars who wrote the ACRRM fellowship examination, $70.6 \%$ passed, while $29.4 \%$ failed the examination. However, passing the ACRRM fellowship examination was not associated with satisfaction with the training environment $\left(\chi^{2}=0.425, \mathrm{df}=1, \mathrm{p}=0.514\right)$.

In terms of preparing for the fellowship examination, $87 \%$ of registrars were satisfied with the pre-examination resources and workshops provided by the training programme. Approximately $90 \%$ of the registrars stated that the examination workshops improved their confidence to undertake the fellowship examination.

\section{Experience of the JCU GPT programme}

Ten registrars (six females and four males) participated in the FGD. Analysis of the qualitative open-ended survey responses and the focus group data revealed four major themes in relation to the participants' experiences of the training programme.

\section{Rich rural/remote educational experience}

The registrars stated that the programme was unique in comparison with training organisations in metropolitan cities. They indicated that the JCU GPT programme was chosen for both the educational experience and the choice of rural GP training.

So if you are in a larger centre there's more specialists and you compete therefore for those opportunities that may in a larger centre be sent off to the specialist care. So one of the main examples of that I could give would be, complex skin cancer operations, which I'd say are quite common in Australia. But depending on where you are, you may not get access to be able to do that, unless you are in a rural centre. (Female 3 FGD).

The participants indicated that there were several advantages of being in their current location, one of which was having greater autonomy.

I think in a more rural or remote area you get a lot more autonomy, whereas if you worked in Brisbane, you know, you wouldn't maybe get tsuch an opportunity (Female 4 FGD)

In addition, training in the region allows the participants to have access to a wide and diverse range of patients.

I think the complexity of patients seen is also much higher than being somewhere more metropolitan. (Male 1 FGD)

Other advantages identified by the registrars were having a sense of community and being able to work in a multidisciplinary team.

Because of the smaller community, you know a lot of the people you're referring to and you're working with, which is always nice. (Female 6 FGD)

\section{Supportive learning environment}

The accessibility and approachability of the training supervisors was also considered as one of the strengths of the programme.

I think approachability too is a big thing that I find. Like we're very much available to our educators and we see them a lot of the time and you can approach them quite easily. Whereas I have colleagues from larger cohorts that they [supervisors] are just a face and not someone that they know and not someone we would feel like we can approach with issues. (Female 1 FGD)

The registrars indicated that they chose their current training location because of the support provided to trainees. They felt the training programme was well structured and supported their learning.

I would still train here. Compared to working at a tertiary hospital in a massive program where there is very little guidance and support. (Male 2 FGD) 
Good experience, well supported and familiarity, and also happy with the place where I live. (Respondent 45 OER)

The participants reported that they received valuable support from their supervisors. They felt the supervisors were easily accessible, respected them and invested in their learning.

Easy approachable and I can knock on anyone's door because it doesn't matter whether it is simple or complex, they're there to help all the time and that I like. (Male 4 FGD)

I think the reciprocal respect that we get from a lot of our supervisors. So they know that we're studying and they treat you with a level of respect for you as a doctor, not just as a trainee, I suppose. I think - I find I really appreciate it because it gives me self-value. (Female 6 FGD)

The registrars also stated that they were satisfied with resources and teaching support provided by the training programme.

I don't know how it's like in other specialties but the availability of resources that are there for us. For example, on GMT, everything's conglomerated there. (Female 5 FGD)

I like regular formal teaching sessions and also the workshops are very good formal teaching sessions. It's also more collegial environment I think because you do get to see people at these workshops face-to-face, and it is collegial as opposed to competing, which seems to be the case in some other specialties, and people are supported. (Female 2 FGD)

In addition, the registrars who passed the fellowship examination stated that the educational support provided helped them to pass their examination and went further to recommend their training post to future registrars.

XXX has provided me with an excellent clinical foundation, which played a large part in helping me pass my exams. I recommend it as a premium training post. (Respondent 337 OER)

I would highly recommend this training post to all new registrars who are seeking a place where they can learn a lot and work in one great, supportive and friendly team. (Female 5 FGD)

Highly recommend this placement for registrars. (Respondent 169 OER)

\section{Readiness to continue with rural practice}

One key attribute of the registrars in relation to their satisfaction with the training environment was the enthusiasm to continue with rural GP. Many participants expressed satisfaction with the training environment and the desire to continue working there.

I would love to work at the XXX hospital in the future. I was very satisfied with this placement. I met a great group of doctors and nurses on this placement. (Respondent 251 OER)

Highly recommend this training post to future registrars. I would like to continue working here. (Male 2 FGD)
XXX is a wonderful practice with supportive medical and other staff. I would highly recommend a placement here for junior and senior registrars as you have the freedom to work in the scope you are comfortable and are given instant assistance or advice from a great range of supervisors when required. I would consider staying at this practice long term. (Respondent 14 OER)

\section{Practice culture}

Despite the positive educational experiences highlighted by the participants, some challenges were identified. The lesser problem was the long hours of commuting to work each day with increasing cost of transportation.

I commute two hour-plus a day, so that cuts into my study time. But otherwise, that's all-that's my only sort of complaint, really. (Male 1 FGD)

The major issue identified by the registrars was referred to as 'practice culture'. The registrars indicated that there were limited number of practices 'that everyone wants to work at (Respondent 162 OER).

I think practices, practice-based. So in xxx there's probably four practices that everyone wants to work at. Then everyone else just has to go to places, because that's where they accept you if you're registrars. So - whereas if you were in a larger city, like Brisbane, obviously, there's a lot more opportunities for work. (Female 1 FGD)

It is believed that the practice and the culture of the practice have an impact on the learning and training of the registrars.

You should be looking in the practices that you're sending registrars to, not just the supervisors you're sending us to, because in a lot of time - and I'm sure you guys agree - the nursing, the practice manager, the other doctors at that practice have a big influence on the kind of education and support you get as well. It doesn't come down to one good doctor or supervisor. (Female 1 FGD).

It was suggested that more support should be provided to registrars who were experiencing difficulties in their training practices.

I really believe that judging from what I've seen other colleagues go through, that there hasn't been enough action taken on those particular ones, when registrars have suffered or that there's been an issue. (Female 2 FGD)

Despite the drawbacks identified, most participants stated that they would choose their current location if given another opportunity to do so. 'Yes, I would choose this location again' (Male 1 FGD).

\section{Triangulation of findings}

The quantitative findings indicated that the participants were satisfied with the educational environment. This was confirmed by the qualitative results, which revealed that the educational environment was unique and provided the registrars with rich learning experiences. Part of 
the gains of the training environment were the sense of community, autonomy and access to a wide range of patients. In addition, the participants believed that the educational environment adequately supported their learning through the provision of learning resources and supervisory support. Furthermore, the qualitative findings revealed that learning support provided was perceived to have been instrumental in passing the fellowship examinations. This outcome was corroborated by the quantitative results that showed that participants who had become fellows expressed higher satisfaction rates with the educational environment compared with those who were still in training. Interestingly, the participants reported a great deal of enthusiasm to continue working in rural and remote practices based on their experience with the educational environment, which has largely been satisfying and supportive. However, the limited number of available practices and the practice culture were considered as some of the potential limitations of GP training in $\mathrm{rural} /$ remote educational environments.

\section{DISCUSSION}

The findings of this mixed methods study suggest that a large proportion of the registrars were highly satisfied with the JCU GPT distributed model. Higher overall satisfaction rate (92\%) was obtained among the registrars in this study in comparison with the reported satisfaction rate $(88 \%)$ in the national survey conducted by the AGPT programme in $2017 .^{32}$ In addition, level of satisfaction was independent of academic performance in the fellowship examinations. This is similar to a previous research that reported no relationship between satisfaction rate and clinical examination scores of medical trainees. ${ }^{33}$ Conversely, another study suggested that trainees who were more satisfied with the educational training environment reported higher performance in their examination. ${ }^{34}$ Although there was no difference in the satisfaction rate by academic performance, however, a large proportion of the participants stated that the resources and pre-examination workshops were facilitated in a collegial environment that aided their preparation for the fellowship examination and boosted their confidence. Evidence suggests that while examination revision activities and resources may be considered useful, satisfaction with the programme is independent of the resources used for examination preparation. ${ }^{35}$

Registrars in this study who had become fellows reported a higher satisfaction rate compared with their counterparts who were still in training. The learning support provided was considered as a major factor that aided passing the fellowship examinations. In addition, the confidence and skills acquired during the training may in part explain the higher satisfaction among the registrars who had become fellows. According to a study conducted by Ayala-Morillas $e t a \hat{l}^{36}$, senior residents were more satisfied with their training compared with the other residents and the higher satisfaction rates were attributed to increased confidence in the skills acquired. Furthermore, previous research have indicated that junior doctors tend to report lower satisfaction rates compared with senior doctors who are specialists or consultants. ${ }^{19}$ Another probable explanation for the reported higher satisfaction rate among registrars who had become fellows may be associated with the feeling of self-actualisation and goal achievement. $^{37}$

The perceived supportive learning environment and the rich rural and remote educational experience provided by the training team were considered as the major reasons for registrar satisfaction with the programme. JCU's distributed GPT model emphasises provision of individualised supervisory support for registrars in rural and remote areas with access to numerous resources, expertise and community-based infrastructure while supporting them to map their training pathway. ${ }^{8}$

The findings of this study address some of the current issues in the literature in relation to satisfaction with work and/or training among GPs. Previous studies have reported that job dissatisfaction was one of the reasons for low retention of GPs. ${ }^{11-15}$ The findings from our study shows that ensuring and providing a supportive learning environment will encourage retention of GPs in rural and remote regions. We have shown that using our unique localised model, providing individualised support for the registrars improved their overall experience and satisfaction level. According to Le Floch et $\mathrm{al}^{17}$, providing GPs with access to professional education aids development of skills, reinforces patient-doctor relationship and improves job satisfaction. Given that retention is dependent on satisfaction, using a localised training programme like ours may attract more GPs to rural and remote areas and improve retention and establishment of GPs in these underserved areas. ${ }^{14} 2038$ Previous evidence suggests that rural pathway training is significantly associated with subsequent rural practice, thereby mitigating the shortage of medical workforce in rural and remote parts of Australia. ${ }^{38}$ Additionally, emphasis should be placed on ensuring good practice culture to increase registrars' satisfaction with the training environment. ${ }^{39}$ Trainees need to feel supported and valued in their work and learning to foster satisfaction and retention. ${ }^{40}$

The high tranportation and training costs due to the remoteness of some training locations were considered challenging. Nonetheless, the participants were not discouraged, possibly because they felt well-supported. This in-turn promotedsatisfaction with the educational environment and motivation to work in the rural/remote locations. ${ }^{20}$ This finding suggests that while there are unavoidable challenges of training in rural and remote regions, having a training model like JCU's distributed model may help to cushion the effect through the provision of individualised support. ${ }^{8}$

\section{Strengths and limitations}

To the best of our knowledge, this study is the first to assess GP registrars' satisfaction with an educational 
environment that uses a distributed training model. In addition, the study used a mixed method approach to explore the perceptions of the registrars about the educational environment. However, the study findings may be cautiously applied to other postgraduate training models.

\section{CONCLUSION}

Understanding the satisfaction of the registrars with the training/educational environment is important for retention and improved patient outcomes in rural and remote Australia. This study suggests that GP registrars undertaking their training with the JCU GPT distributed model are satisfied with the training environment, and their satisfaction is independent of their academic performance. However, ensuring a supportive work environment is needed to increase satisfaction with the educational environment and enhance retention of GPs in rural practice.

\section{Twitter Bunmi S. Malau-Aduli @bmalauaduli and Faith 0. Alele @D Faith}

Acknowledgements The authors would like to acknowledge all the James Cook University (JCU) GP registrars who participated in the study. The JCU General Practice Training administrative staff are appreciated for facilitating the data collection process.

Contributors BSM-A, PH, P-AT and CR designed the study. BSM-A supervised the project and oversaw the project administration. PH and CR coordinated the survey and focus group discussion. BSM-A and FOA conducted the statistical and qualitative data analysis. BM-A and FOA wrote the original draft of the manuscript. All authors reviewed and approved the final version of the manuscript.

Funding The authors have not declared a specific grant for this research from any funding agency in the public, commercial or not-for-profit sectors.

Map disclaimer The depiction of boundaries on this map does not imply the expression of any opinion whatsoever on the part of BMJ (or any member of its group) concerning the legal status of any country, territory, jurisdiction or area or of its authorities. This map is provided without any warranty of any kind, either express or implied.

Competing interests None declared.

Patient and public involvement statement Patients and the public were not involved in the planning or design of this study.

Patient consent for publication Not required.

Ethics approval Ethics approval for the study was obtained from the JCU Human Research Ethics Committee (H6771). Written consent was obtained from study participants.

Provenance and peer review Not commissioned; externally peer reviewed.

Data availability statement The data relevant to this study are included in the artcle.

Supplemental material This content has been supplied by the author(s). It has not been vetted by BMJ Publishing Group Limited (BMJ) and may not have been peer-reviewed. Any opinions or recommendations discussed are solely those of the author(s) and are not endorsed by BMJ. BMJ disclaims all liability and responsibility arising from any reliance placed on the content. Where the content includes any translated material, BMJ does not warrant the accuracy and reliability of the translations (including but not limited to local regulations, clinical guidelines, terminology, drug names and drug dosages), and is not responsible for any error and/or omissions arising from translation and adaptation or otherwise.

Open access This is an open access article distributed in accordance with the Creative Commons Attribution Non Commercial (CC BY-NC 4.0) license, which permits others to distribute, remix, adapt, build upon this work non-commercially, and license their derivative works on different terms, provided the original work is properly cited, appropriate credit is given, any changes made indicated, and the use is non-commercial. See: http://creativecommons.org/licenses/by-nc/4.0/.

\section{ORCID iDs}

Bunmi S. Malau-Aduli http://orcid.org/0000-0001-6054-8498

Faith 0. Alele http://orcid.org/0000-0001-5570-3641

\section{REFERENCES}

1 Starfield B, Shi L, Macinko J. Contribution of primary care to health systems and health. Milbank Q 2005;83:457-502.

2 Zhao Y, Russell DJ, Guthridge S, et al. Long-Term trends in supply and sustainability of the health workforce in remote Aboriginal communities in the Northern Territory of Australia. BMC Health Serv Res 2017;17:836.

3 Anderson I, Robson B, Connolly M, et al. Indigenous and tribal peoples' health (the Lancet-Lowitja Institute global collaboration): a population study. Lancet 2016;388:131-57.

4 Smith KB, Humphreys JS, Wilson MGA. Addressing the health disadvantage of rural populations: how does epidemiological evidence inform rural health policies and research? Aust J Rural Health 2008;16:56-66.

5 Wakerman J, Humphreys JS. Sustainable workforce and sustainable health systems for rural and remote Australia. Med J Aust 2013;199:14-17.

6 Emery JD, Skinner LP, Morgan S, et al. Future models of general practice training in Australia. Med J Aust 2011;194:S97.

7 Wearne SM, Magin PJ, Spike NA. Preparation for general practice vocational training: time for a rethink. Med J Aust 2018;209:52-4.

8 James Cook University. General practice training, 2016. Available: https://www.gmt.edu.au/ [Accessed 14 Feb 2019].

9 Buddeberg-Fischer B, Dietz C, Klaghofer R, et al. Swiss residents' arguments for and against a career in medicine. BMC Health Serv Res 2006;6:98.

10 Westerman JW, Nowicki MD, Plante D. Fit in the classroom: predictors of student performance and satisfaction in management education. J Manag Educ 2002;26:5-18.

11 Malhotra J, Wong E, Thind A. Canadian family physician job satisfaction - is it changing in an evolving practice environment? An analysis of the 2013 National Physician Survey database. BMC Fam Pract 2018;19:100.

12 Van Ham I, Verhoeven AAH, Groenier KH, et al. Job satisfaction among general practitioners: a systematic literature review. Eur $J$ Gen Pract 2006;12:174-80.

13 Le Floch B, Bastiaens H, Le Reste JY, et al. Which positive factors determine the GP satisfaction in clinical practice? A systematic literature review. BMC Fam Pract 2016;17:133.

14 Marchand C, Peckham S. Addressing the crisis of GP recruitment and retention: a systematic review. Br J Gen Pract 2017;67:e227-37.

15 Owen K, Hopkins T, Shortland T, et al. Gp retention in the UK: a worsening crisis. findings from a cross-sectional survey. BMJ Open 2019;9:e026048.

16 Lee DM, Nichols T. Physician recruitment and retention in rural and underserved areas. Int J Health Care Qual Assur 2014;27:642-52.

17 Le Floch B, Bastiaens H, Le Reste JY, et al. Which positive factors give general practitioners job satisfaction and make general practice a rewarding career? a European multicentric qualitative research by the European general practice research network. BMC Fam Pract 2019;20:96.

18 Ulmer B, Harris M. Australian GPs are satisfied with their job: even more so in rural areas. Fam Pract 2002;19:300-3.

19 Joyce CM, Schurer S, Scott A, et al. Australian doctors' satisfaction with their work: results from the MABEL longitudinal survey of doctors. Med J Aust 2011;194:30-3.

20 McGrail MR, Humphreys JS, Scott A, et al. Professional satisfaction in general practice: does it vary by size of community? Med J Aust 2010;193:94-8

21 Behmann M, Schmiemann G, Lingner H, et al. Job satisfaction among primary care physicians: results of a survey. Dtsch Arztebl Int 2012:109:193.

22 Malau-Aduli BS, Smith AM, Young L, et al. To stay or go? unpacking the decision-making process and coping strategies of international medical graduates practising in rural, remote, and regional Queensland, Australia. PLoS One 2020;15:e0234620.

23 Peel R, Young L, Reeve C, et al. The impact of localised general practice training on Queensland's rural and remote general practice workforce. BMC Med Educ 2020;20:119.

24 Tashakkori A, Teddlie C, Teddlie CB. Mixed methodology: combining qualitative and quantitative approaches. Sage, 2008.

25 Kaushik V, Walsh CA. Pragmatism as a research paradigm and its implications for social work research. Soc Sci 2019;8:255. 
26 Ivankova NV, Creswell JW, Stick SL. Using mixed-methods sequential explanatory design: from theory to practice. Field methods 2006;18:3-20.

27 Kirkpatrick JD, Kirkpatrick WD. Kirkpatrick's four levels of training evaluation. Association for Talent Development, 2016.

28 Malau-Aduli BS, Alele F, Collares CF, et al. Validity of the scan of postgraduate educational environment domains (speed) questionnaire in a rural general practice training setting. BMC Med Educ 2019;19:25.

29 O'Leary Z. The essential guide to doing your research project. 3rd ed. London, United Kingdom; Thousand Oaks, California: SAGE Publications, 2017.

30 Braun V, Clarke V. Using thematic analysis in psychology. Qual Res Psychol 2006;3:77-101.

31 O'Brien BC, Harris IB, Beckman TJ, et al. Standards for reporting qualitative research: a synthesis of recommendations. Acad Med 2014;89:1245-51.

32 Taylor R, Clarke L, Edwards D. Australian general practice training program national report on the 2018 national registrar survey, 2019.

33 Chinthammitr Y, Chinthamitr Y, Chierakul N. Learning environment and resident achievement. J Med Assoc Thai 2014:97:1269-73.
34 Shimizu T, Tsugawa Y, Tanoue Y, et al. The hospital educational environment and performance of residents in the general medicine in-training examination: a multicenter study in Japan. Int J Gen Med 2013;6:637-40.

35 Swift M, Spake E, Gajewski BJ. Student performance and satisfaction for a musculoskeletal objective structured clinical examination. J Allied Health 2013;42:214-22.

36 Ayala-Morillas LE, Fuentes-Ferrer ME, Sánchez-Díaz J. Factors associated with residents' satisfaction with their training as specialists. Revista Clínica Española 2014;214:175-83.

37 Wach F-S, Karbach J, Ruffing S, et al. University students satisfaction with their academic studies: personality and motivation matter. Front Psychol 2016;7:55.

38 McGrail MR, Russell DJ, Campbell DG. Vocational training of genera practitioners in rural locations is critical for the Australian rural medical workforce. Med J Aust 2016;205:216-21.

39 Gregory S, Demartini C. Satisfaction of doctors with their training: evidence from UK. BMC Health Serv Res 2017;17:851.

40 Buddeberg-Fischer B, Dietz C, Klaghofer R. Swiss residents' arguments for and against a career in medicine. BMC Health Serv Res 2006;6:98. 